\title{
UDP Performance Analysis of IPv4 and IPv6 on Windows Vista and Windows XP over Fast Ethernet Peer-Peer LAN
}

\author{
Chris Manford, Samad S. Kolahi, Burjiz. K. Soorty, Zhang Qu, and Navneet Chand \\ UNITEC New Zealand \\ cmanford@unitec.ac.nz \\ burjizsoorty@hotmail.com
}

\begin{abstract}
In this paper, the performance of IPv4 and IPv6 are compared using Windows Vista and the UDP (User Datagram Protocol) throughput results are compared with Windows XP in a peer-peer environment. The results indicated that the UDP bandwidth using Windows Vista and fast Ethernet for IPv4 ranged between 26.5 and 85.6 Mbps and between 24 and 84.6 Mbps for IPv6. Windows XP bandwidth ranged from 21.8 and 86 Mbps for IPv4 and between 20.7 and 81 Mbps for IPv6.
\end{abstract}

Keyword: Performance Evaluation, IPv4, IPv6, Fast Ethernet Peer-Peer LAN, Windows XP and Vista.

\section{INTRODUCTION}

The growth in internet has significantly increased the number of internet addresses required. As number of IPv4 are limited and exhausting, this is successfully overcome by IPv6 which supports a total of $2^{128}$ addresses as opposed to $2^{32}$ for IPv4. As the transition to IPv6 gets closer, newer operating systems aim to put a higher emphasis into enhancing IPv6 performance. Several works have been carried out on evaluating IPv4 and IPv6 that have shown their performance to vary largely depending on the operating system used on the network [1].

Vista is a new version of Windows operating system and was introduced to replace Windows XP. In this paper, the UDP performance of IP4 against IP6 is carried out for Windows Vista and the results compared with Windows XP.

The previous research work on IP4 and IP6 comparison are as follows. In 1998, Draves et al. [2] conducted a throughput evaluation of IPv6 using Windows NT over a network where two clients were directly connected through a crossover cable. Their study ran TCP throughput tests for both IPv4 and IPv6 over $10 \mathrm{Mb} / \mathrm{s}$ and $100 \mathrm{Mb} / \mathrm{s}$ Ethernet. The analysis however did not consider any other additional protocols such as UDP or any parameters such as different packet sizes that could potentially affect network performance. Their findings showed IPv6 has 1.9\% less throughput compared to the IPv4.

In 2000, Ariga et al. [3] conducted a performance evaluation of data transmission through IPv6 and IPv4 using IPSec on a network implementing the Unix-like open source operating system called FreeBSD. The authors took into account the TCP and UDP protocols however did not consider the different packet sizes. Their studies concluded that the TCP/UDP throughput for IPv6 was almost equivalent to that of IPv4.

In 2003, Zeadally and Raicu [4] conducted a performance evaluation of IPv4 and IPv6 on Windows 2000 and Solaris 8. Two identical workstations were connected using a point-topoint link in-order to eliminate variables such as router latency from the experiments. This study included an additional metric of RTT (Round Trip Time) along with the TCP/UDP throughput. Their analysis concluded that the performance of IPv4 and IPv6 varied significantly, especially a notably large difference in throughput for packet sizes smaller than 256 bytes in Solaris. The difference, an apparent three times higher throughput for IPv4 mainly due to the rather large increase in socket-creation and connection time for IPv6 because of its high overhead [4].

In 2004, Zeadally et al. [1] conducted another empirical performance evaluation of IPv4 and IPv6 protocol stack. They used Linux as the operating system and compared the results to their 2003 study on Windows 2000 and Solaris 8. Throughput and RTT were measured for TCP and UDP protocols. Their evaluation concluded that both IPv4 and IPv6 on Linux outperformed Windows 2000 and Solaris 8 for both the metrics used, the latter having a lower margin of difference compared to Windows 2000. In 2006, Mohammed et al. [5] carried out an evaluation on the performance of IPv6 on two different operating systems, namely, Windows 2003 and Linux based Red Hat 9. The study implemented three different test beds each representing two different environments. The first, a "Normal View Environment" where the payload size was less than the MTU (Maximum Transmission Unit) and the second, a "Global View Environment" where the payload size was greater than the MTU. The MTU can best be defined as the highest amount of data that can be transferred in one physical frame on the network. Their studies concluded that the performance of IPv6 was far better in Red Hat 9 than in Windows Server 2003 as the latter fragmented packets larger than 1440 bytes as opposed to the former where fragmentation occurred at 16384 bytes.

In 2008, Narayan et al. [6] carried out a performance comparison of IPv4 and IPv6 using Windows XP operating 
system over a network where two clients were directly connected through a standard Category 5e crossover cable. The study performed an evaluation on IPv4 and IPv6 for TCP and UDP protocols by measuring throughput and RTT for various packet sizes. Their research concluded that IPv6 performed better for TCP and IPv4 performed better for UDP with Windows XP.

There has been no work done to date on Windows Vista. The lack of available research on IPv4 and IPv6 performance on Windows Vista led to the main motivation behind this work. The contribution of this paper is to compare the UDP performance of IPv4 and IPv6 on Windows Vista in peer-peer environment. We studied the UDP throughput and round trip time for IPv4 and IPv6 on a range of packet sizes varying from 128 to 1408 bytes. The results are then compared to that of IPv4 and IPv6 performance on Windows XP reported in [6].

The organization of this paper is as follows. In the next section the network setup is discussed. Section three covers information regarding the data generation and traffic measurement tool. Section four covers the results and the last sections include the conclusion followed by the references.

\section{NETWORK SETUP}

The proposed network test-bed (figure 1) was setup through a direct connection via standard Category 5e cabling between two workstations. This was done in order to calculate the raw throughput and RTT without the use of a hub, switch or a router. It was also done so as to maintain consistency with similar research shown in the past including the previous work done on Windows XP [6]. The computer hardware comprised of an Intel® Core ${ }^{\mathrm{TM}} 2$ Duo $63001.87 \mathrm{GHz}$ processor with 2.00 GB RAM for the efficient operation of Windows Vista, an Intel Pro/100 S Desktop Adapter NIC and a Western Digital Caviar SE 160 GB hard-drive on the two workstations.

The operating system installed was Microsoft Windows Vista (plus Service Pack 1).

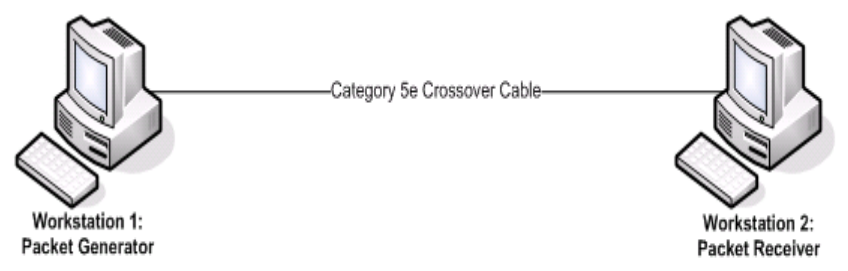

Figure 1: Network Test-Bed

\section{Data Generation AND TRAFFiC MeAsurement ToOL}

IP Traffic [7] was selected as the traffic generating and measurement tool for its compatibility with Windows Vista, and for its powerful analysis of a wide range of quality of service parameters to acquire accurate results. Most importantly it was chosen in order to keep consistency with the results produced for IPv4 and IPv6 performance on Windows XP [6]. Furthermore, IP Traffic has been used extensively for various other research including performance evaluation of network security [8] and impact of encryption effects on network performance [9].

\section{RESULTS}

The UDP throughput and RTT were measured for both IPv4 and IPv6 for various packet sizes. The range of packet sizes varied from 128 to 1408 bytes over a Peer to Peer network running Windows Vista operating system.

The experiments comprised of performing 40 test runs for each specific packet size (128 to 1408 bytes). One run included sending one million packets of the particular packet size and protocol. The results were then averaged and the standard deviation of results recorded.

\section{UDP Throughput Comparison for IPv4 and IPv6}

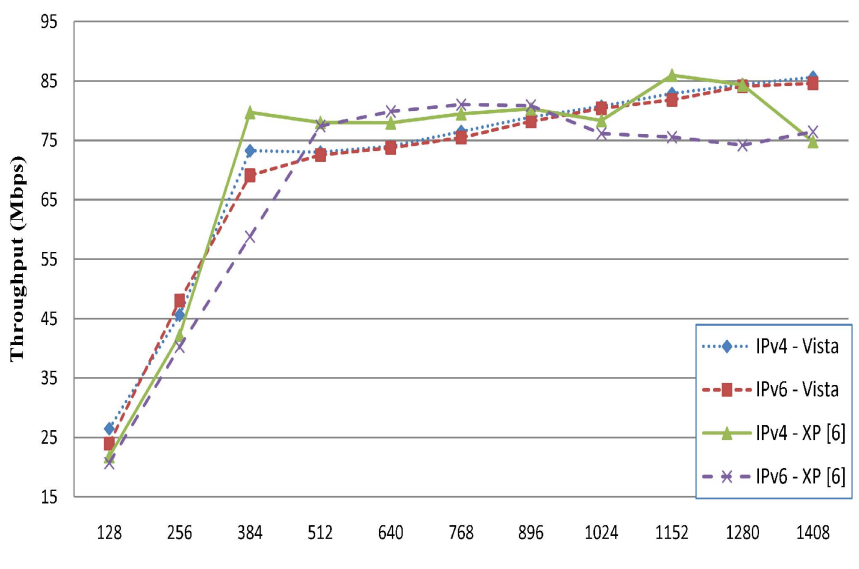

\section{Figure 2: UDP Throughput Comparison for IPv4 and IPv6 on Windows Vista vs. Windows XP}

Figure 2 shows the UDP throughput for IPv4 and IPv6 on Windows XP [6] and Vista. As depicted for Windows Vista, a rapid increase in throughput is observed with the lower range of packet size 128-384 bytes for IPv4 and 128-512 bytes for IPv6 before the growth rate reduces on the remaining packet sizes. The difference in throughput between IPv4 and IPv6 performance is barely noticeable, overall results show UDP throughput is slightly higher on IPv4 although that difference is by far insignificant.

Windows XP results in [6] showed UDP throughput was slightly higher on IPv4 than on IPv6 with a maximum difference of $21 \mathrm{Mbps}$ for packet size 384 bytes (IPv4 bandwidth was $79.8 \mathrm{Mbps}$ while IPv6 was at $58.8 \mathrm{Mbps}$ ). For packet size of 1152, IPv4 bandwidth was 86 Mbps while IPv6 was at $75.6 \mathrm{Mbps}$. For most packet sizes, the UDP results showed an insignificant difference between IPv4 and IPv6, however, UDP throughput was marginally higher on IPv4 but for some packet sizes IPv6 gives higher bandwidth (Figure 2).

Windows XP results show a high inconsistency in UDP bandwidth for various packet sizes, for instance with IPv6, performance is lower on XP than Vista for packet sizes 128 to 
384 and 1024 to 1408 but higher for packet sizes 512 to 896 . On Windows Vista, IPv4 and IPv6 UDP throughput show a far more stable performance with a steady increase in throughput as packet size increases. It is hard to determine which OS gives a higher throughput for IPv4 as both OS's show a drop in bandwidth on about four packet sizes. Compared to Vista however throughput on XP fluctuates with either a drop or an increase in bandwidth with increasing packet sizes. The standard deviation for the throughput results are recorded in Table 1. The UDP bandwidth range using Windows Vista for fast Ethernet with IPv4 was between 26.5 and 85.6 Mbps and between 24 and $84.6 \mathrm{Mbps}$ for IPv6. Windows XP bandwidth ranged from 21.8 and $86 \mathrm{Mbps}$ for IPv4 and between 20.7 and 81 Mbps for IPv6.

TABLE I

STANDARD DEVIATION FOR THROUGHPUT

\begin{tabular}{|r|l|l|l|l|}
\hline & \multicolumn{2}{|c|}{ Vista } & \multicolumn{2}{c|}{ XP } \\
\hline Bytes & IPv4 & IPv6 & IPv4 & IPv6 \\
\hline 128 & 0.43 & 0.46 & 0.26 & 0.1 \\
\hline 256 & 0.44 & 0.88 & 0.67 & 0.28 \\
\hline 384 & 1.06 & 1.5 & 0.75 & 0.55 \\
\hline 512 & 1.27 & 0.94 & 0.81 & 0.53 \\
\hline 640 & 0.54 & 0.71 & 1.01 & 0.52 \\
\hline 768 & 0.65 & 0.62 & 0.61 & 0.55 \\
\hline 896 & 0.97 & 0.34 & 1.27 & 1.05 \\
\hline 1024 & 0.85 & 0.6 & 0.44 & 0.82 \\
\hline 1152 & 0.67 & 0.55 & 0.69 & 0.71 \\
\hline 1280 & 0.48 & 0.67 & 3.16 & 0.8 \\
\hline 1408 & 0.38 & 0.38 & 1.21 & 0.85 \\
\hline
\end{tabular}

Considering UDP throughput for IPv6, Windows Vista performed comparatively better than XP with the maximum difference of 10.3 Mbps (58.8 Mbps for XP vs 69.1 Mbps for Vista) for 384 byte packet size. UDP throughput for IPv4 resulted with similar performance for both operating systems as Windows Vista showed a higher throughput for packet sizes 128, 256, 1024 and 1280 bytes whereas Windows XP [6] showed a higher throughput for packet sizes $384,513,640,768$ and 1024 bytes.

The results produced in XP [6] also showed a plunge in UDP throughput for IPv4 on the packet size of 1408 bytes. This is inconsistent with our results produced for Windows Vista and also with results produced for Windows 2000, Solaris and Linux in [1] by Zeadally et al. where throughput steadily grows up to 1408 bytes. The gain in UDP throughput as the packet size increases is likely due to the amortization of overheads associated with larger user packet sizes (larger user payloads) [4].

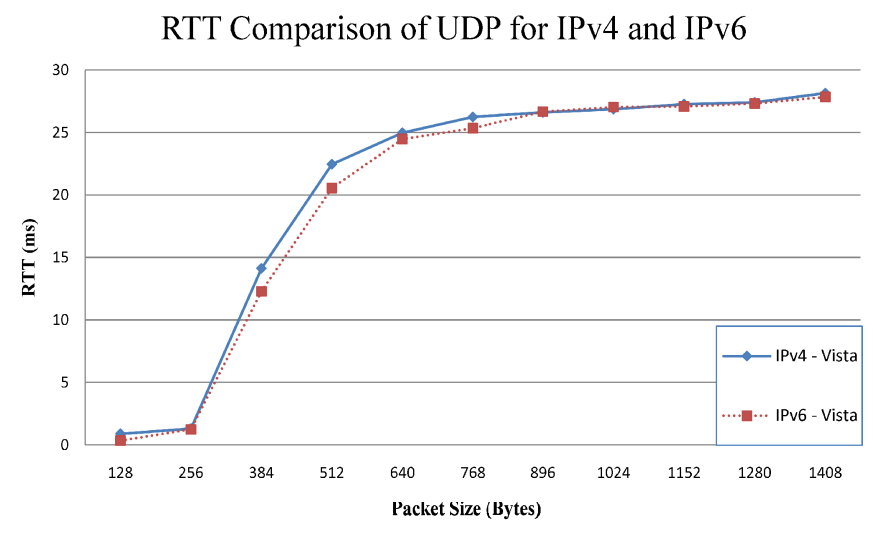

Figure 3: RTT Comparison of UDP for IPv4 and IPv6 on Windows Vista

Figure 3 shows the UDP round trip time for IPv4 and IPv6 on Windows Vista. No data is available on RTT for XP in [6]. The UDP results show a gain in delay for IPv4 and IPv6 as each packet size increases.

TABLE II

STANDARD DEVIATION FOR RTT

\begin{tabular}{|c|c|c|}
\hline & \multicolumn{2}{|c|}{ Vista } \\
\hline Bytes & IPv4 & IPv6 \\
\hline 128 & 0.08 & 0.15 \\
\hline 256 & 0.2 & 0.25 \\
\hline 384 & 1.33 & 1.26 \\
\hline 512 & 1.18 & 2.06 \\
\hline 640 & 0.4 & 0.73 \\
\hline 768 & 0.33 & 0.55 \\
\hline 896 & 0.18 & 0.5 \\
\hline 1024 & 0.11 & 0.14 \\
\hline 1152 & 0.24 & 0.05 \\
\hline 1280 & 0.21 & 0.12 \\
\hline 1408 & 0.33 & 0.11 \\
\hline
\end{tabular}

The RTT for IPv4 ranged from $0.9 \mathrm{~ms}$ to $28.2 \mathrm{~ms}$ and for IPv6 ranged from $0.3 \mathrm{~ms}$ to $27.8 \mathrm{~ms}$. The UDP round trip time for IPv4 and IPv6 appear very close. The average RTT is slightly lower on IPv6 than on IPv4 for packet sizes 384-640 bytes. The highest point of difference stands at packet size 384 bytes where IPv6 has a RTT of $12.3 \mathrm{~ms}$ comapred to $14.1 \mathrm{~ms}$ of IPv4. The standard deviation for the above RTT results are recorded in the Table 2.

The increase in delay with the increase in each packet size is likely due to the amortization of overheads associated with larger packet sizes (larger user payloads) thus higher transmission time [4]. UDP does not use any form of error correction and no acknowlegenment is sent back to the source partly due to it being a connectionless protocol. Thereofore, the RTT results for UTP has insignificant difference between IPv4 and IPv6. 


\section{CONCLUSION}

In this study, we compared the UDP performance of IPv4 with IPv6 for both Windows Vista and Windows XP. The results indicate that generally as the packet size increases, the bandwidth increases. In Vista, the difference in UDP throughput between IPv4 and IPv6 performance is barely noticeable, overall results show UDP throughput is slightly higher on IPv4 although that difference is by far insignificant. Using IPv6, Windows Vista performed comparatively better than XP with the maximum difference of 10.3 Mbps (58.8 Mbps for XP vs 69.1 Mbps for Vista) for 384 byte packet size. UDP throughput for IPv4 resulted with similar performance for both operating systems as Windows Vista showed a higher throughput for packet sizes 128, 256, 1024 and 1280 bytes whereas Windows XP showed a higher throughput for packet sizes 384, 513, 640, 768 and 1024 bytes. In Vista, the round trip time for IPv4 ranged from $0.9 \mathrm{~ms}$ to $28.2 \mathrm{~ms}$ and for IPv6 IPv6 ranged from $0.3 \mathrm{~ms}$ to $27.8 \mathrm{~ms}$. The UDP Round Trip Time for IPv4 and IPv6 appear very close.

\section{FUTURE WORKS}

Future works include performing and comparing an evaluation of IPv4 and IPv6 using different network operating systems environments; Windows Server 2008 and Linux and using Windows 7 as client operating systems over Gigabit Ethernet LAN's.

\section{ACKNOWLEDGMENT}

The authors would like to thank Unitec Institute of Technology for funding the research team and providing the inventory needed.

\section{REFERENCES}

[1] S. Zeadally, R. Wasseem, and I. Raicu.: 'Comparison of end-system IPv6 protocol stacks', IEE Proceedings Communications, 2004, 151, (3), pp. 238-242.

[2] R.P. Draves, A. Mankin, and B.D. Zill, "Implementing IPv6 for Windows NT," Proc. Proceedings, of the 2nd USENIX Windows NT Symposium, 1998.

[3] S. Ariga, K. Nagahashi, M. Minami et al., "Performance evaluation of data transmission using IPSec over IPv6 networks," Proc. Proceedings of the INET, 2000.

[4] S. Zeadally and L. Raicu, "Evaluating IPv6 on Windows and Solaris," Internet Computing, IEEE, vol. 7, no. 3, 2003, pp. 51-57.

[5] S.S. Mohamed, A.Y.M. Abusin, and D. Chieng., "Evaluation of IPv6 and comparative study with different operating systems," Proc. Third International Conference on Information Technology and Applications, 2005, pp. 665-670 vol.662.

[6] S. Narayan, S.S. Kolahi, Y. Sunarto, D.D.T. Nguyen, and P. Mani, "Performance comparison of IPv4 and IPv6 on various windows operating systems," Proc. 11th International Conference on Computer and Information Technology, 2008, pp. 663-668.

[7] ZTI Telecom, "IP Traffic - test \& measure," http://www.zti-telecom.com.

[8] N. Baghaei and R. Hunt, "IEEE 802.11 wireless LAN security performance using multiple clients," Proc. Proceedings, The 12th IEEE International Conference on Networks, 2004, pp. 299-303 vol.291.

[9] B. Ezedin, M. Boulmalf, A. Alteniji, H.A. Suwaidi et al., "Impact of Security on the Performance of Wireless-Local Area Networks," Proc. Innovations in Information Technology, 2006, pp. 1-5. 